\title{
Dual effect of structure and hydration on magnesium-ion insertion into
}

electrodeposited $\mathrm{V}_{2} \mathrm{O}_{5}$ thin films

Brandon Johnston*, Hakeem Henry*, Emily Sahadeo*, Darrin Liau*, and Sang Bok Lee* *Department of Chemistry and Biochemistry

\section{Introduction}

As global energy needs increase, there is a growing demand for safe, the current state of the art in battery technology, are beginning to reach their limits in terms of the amount of energy density they can offer. Magnesium-ion batteries show promise as potential candidates to fill this demand as a result of their large energy density and specific volumetric capacity. When compared with conventional ion battery technologies, such as lithium-ion batteries, the raw materials for magnesium ion batteries are more abundant, and they are much safer. However, a few significant challenges have slowed progress in magnesium ion battery research; such as poor magnesium-ion insertion reversibility at the cathode interface, passivation at the anode surface, and poor compatibility with
electrolyte solvents ${ }^{1}$.

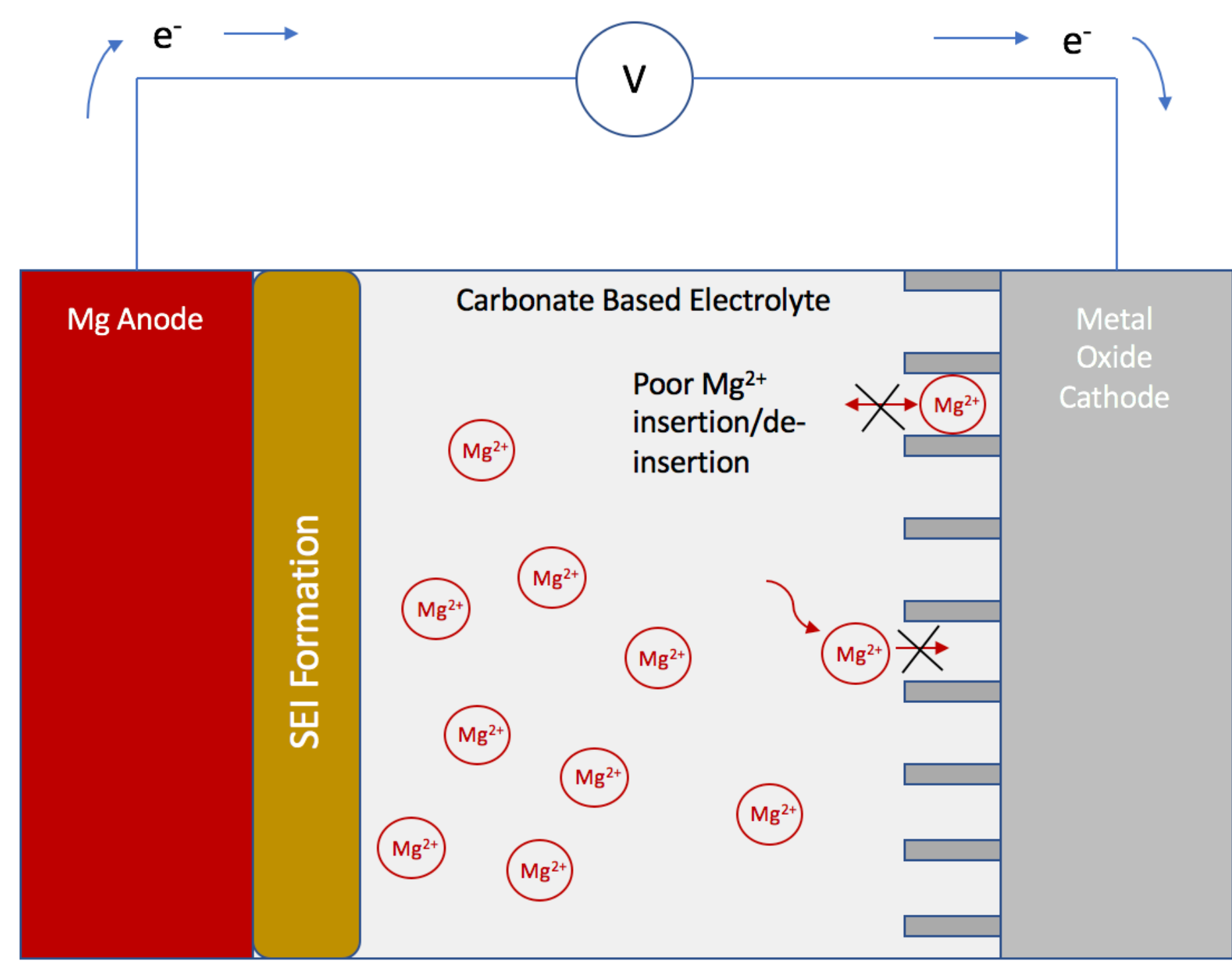

Schematic of Standard Mg-ion battery

\section{Cathodes}

Mg batteries suffer from slow reaction kinetics at the cathode interface due to strong electrostatic repulsion attributable to the bivalence of the $\mathrm{Mg}$ ion. As a result, we chose to focus on improving Mg-ion insertion/de-insertion kinetics by enhancing the performance of $\mathrm{V}_{2} \mathrm{O}_{5}$, a well studied metal oxide redox active material for $\mathrm{Mg}$ ion batteries.

Vanadium (V) Oxide is a popular cathode material candidate for magnesium-ion batteries owing to its high stability, operating voltage, and specific capacity in its crystal form ${ }^{1}$. However, new research suggests that amorphous $\mathrm{V} 2 \mathrm{O} 5$ cathodes might offer greater capacity for Mg-lon insertion owing to their large interlayer spacing. In this work, we evaluate this claim by

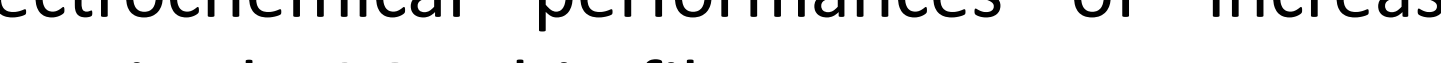

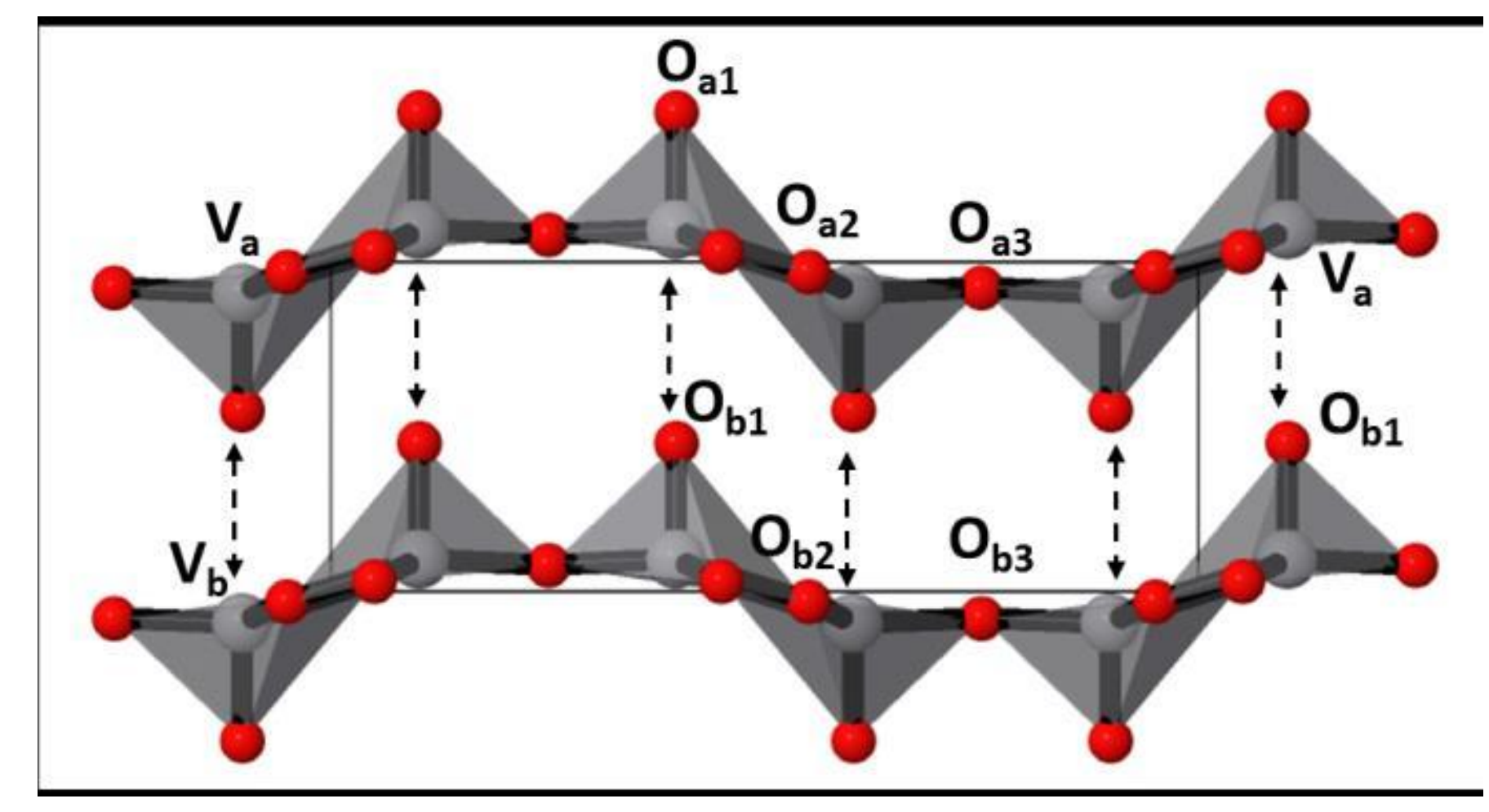

Crystal lattice structure of $\mathrm{V}_{2} \mathrm{O}_{5}$

\section{Cathode Development}

\section{$\mathrm{V}_{2} \mathrm{O}_{5}$ Thin Film Formation}

$\mathrm{V}_{2} \mathrm{O}_{5}$ was used as the high capacity redox-active material for $\mathrm{Mg}$-ion storage. Thin film cathodes were synthesized potentiostatically using a stainless steel spacer template. By holding a constant voltage of $1.5 \mathrm{~V} \mathrm{vs}$. Ag/AgCl in a solution containing $0.25 \mathrm{M}$ vanadyl sulfate, $\mathrm{V}_{2} \mathrm{O}_{5}$ was deposited onto the surface of the stainless steel Samples were then anneled in air at 200,300, or $500 \mathrm{C}$ for 3 hours. Amorphous samples were not annealed.

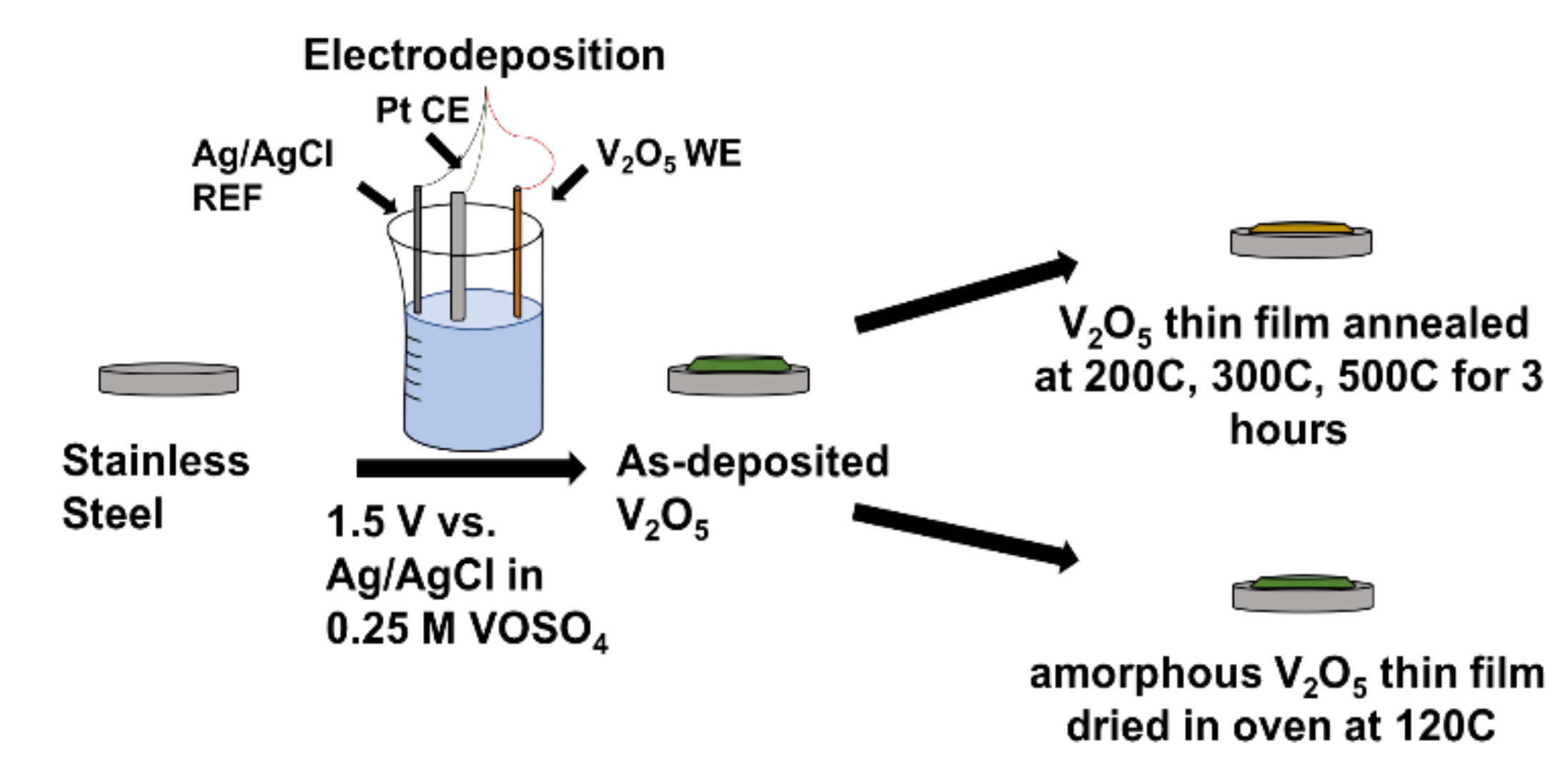

Schematic of the electrodeposition and annealing of the $\mathrm{V}_{2} \mathrm{O}_{5}$ films.

\section{Characterization of Crystallinity}



Raman spectroscopy data for V205 thin films annealed at increasing temperature. A clear transition to long-range crystalline $\mathrm{V} 2 \mathrm{O} 5$ can be seen when samples are annealed temperatures greater than 300C. A-V2O5 stands for amorphous V2O5.

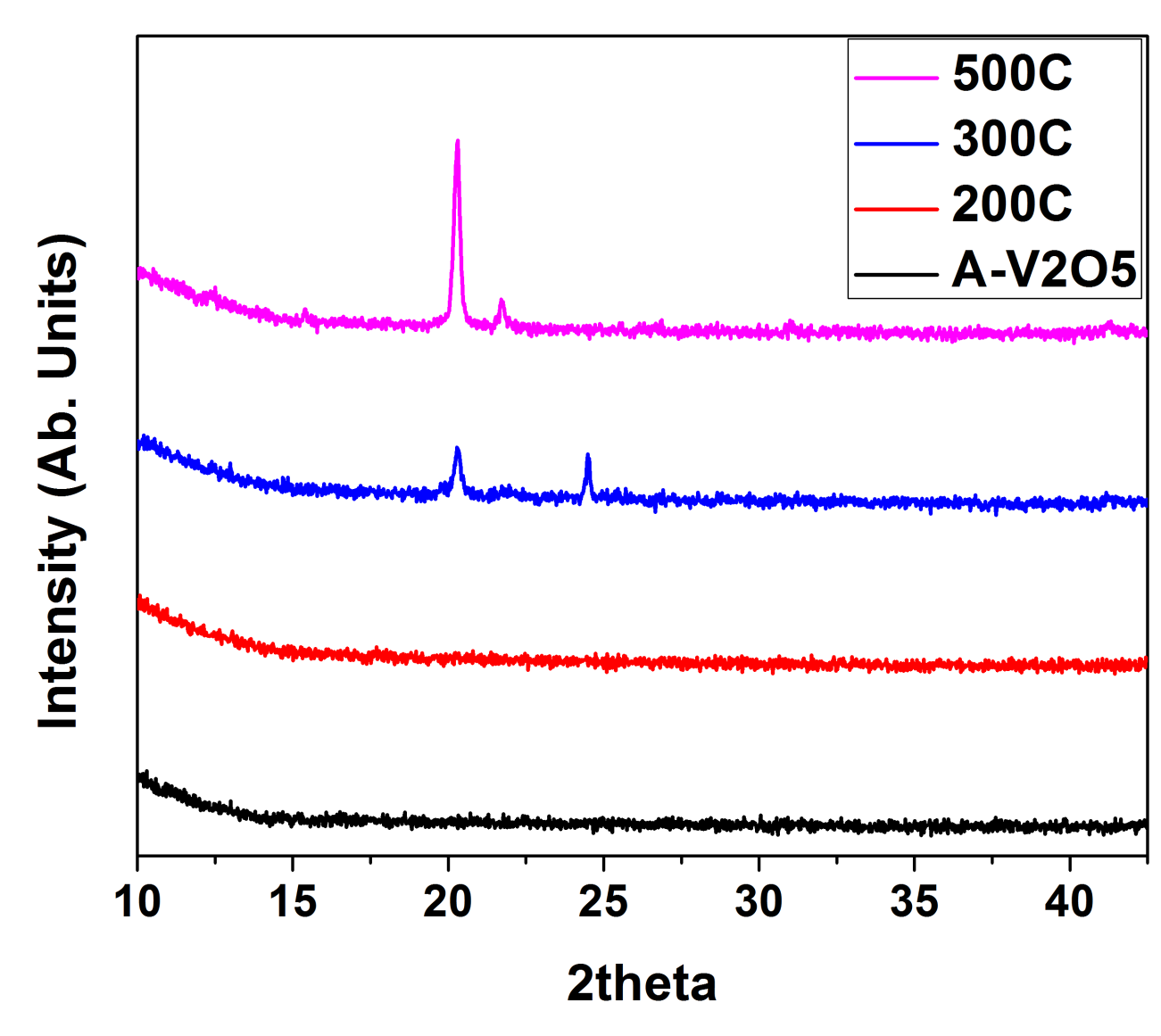

RD data for V2O5 thin films annealed at increasing temperature. A clear transition to long-range crystalline V205 can be seen when samples are annealed at temperatures greater than $300 \mathrm{C}$ by the emergence of peaks at $20^{\circ}, 22^{\circ}$, and $24^{\circ}$. A-V205 stands for amorphous $\mathrm{V} 205$.

\section{Electrochemical Analysis}

CV and Charge-Discharge Cycling Performance
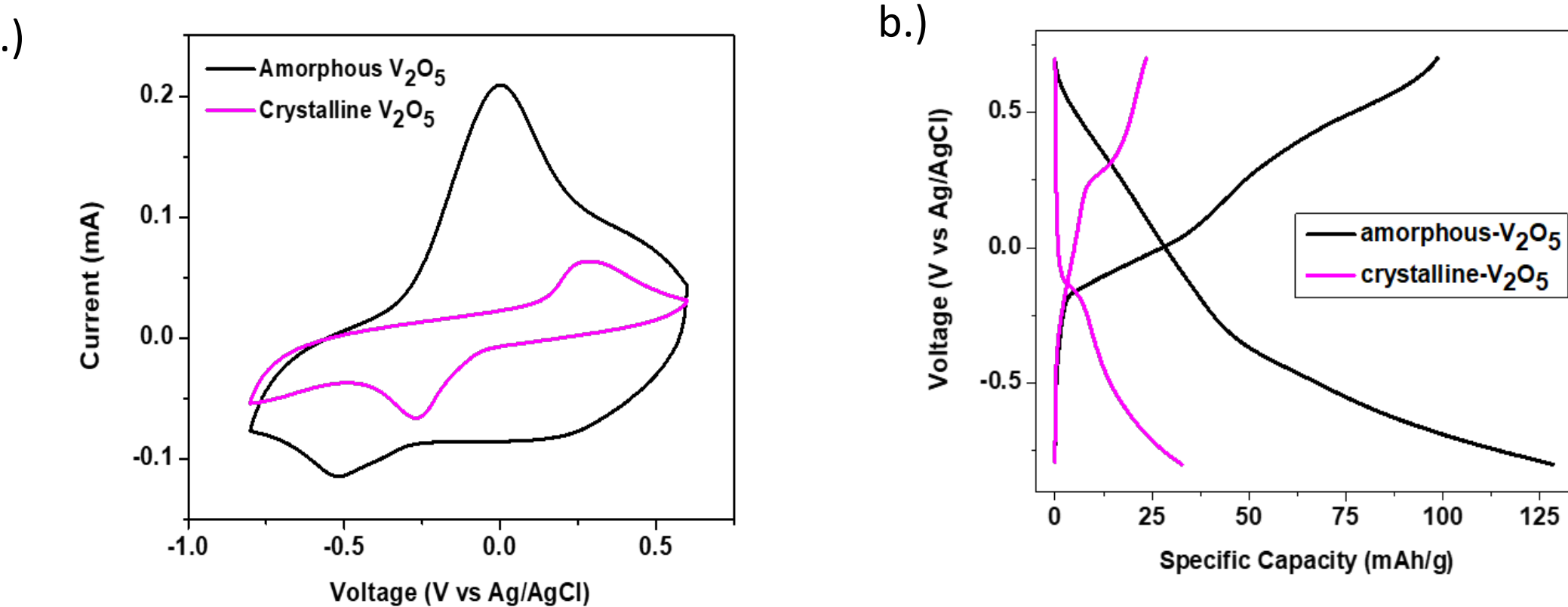

a.) Rate Performance Data for Amorphous and Crystalline V2O5 (500C) cycled in $0.1 \mathrm{M} \mathrm{Mg}\left(\mathrm{ClO}_{4}\right)_{2}{ }_{2}^{*} 6 \mathrm{H}_{2} \mathrm{O}$. Amorphous $\mathrm{V} 2 \mathrm{O} 5$ outperforms crystalline 205 ( $500 \mathrm{C}$ ) at every current density. b.) anst 105 at low curent density. It should be nous and $500 \mathrm{c}$ a amorphous 205 samples did not go through a rigorous ding protocol.

\section{Rate Performance Data}

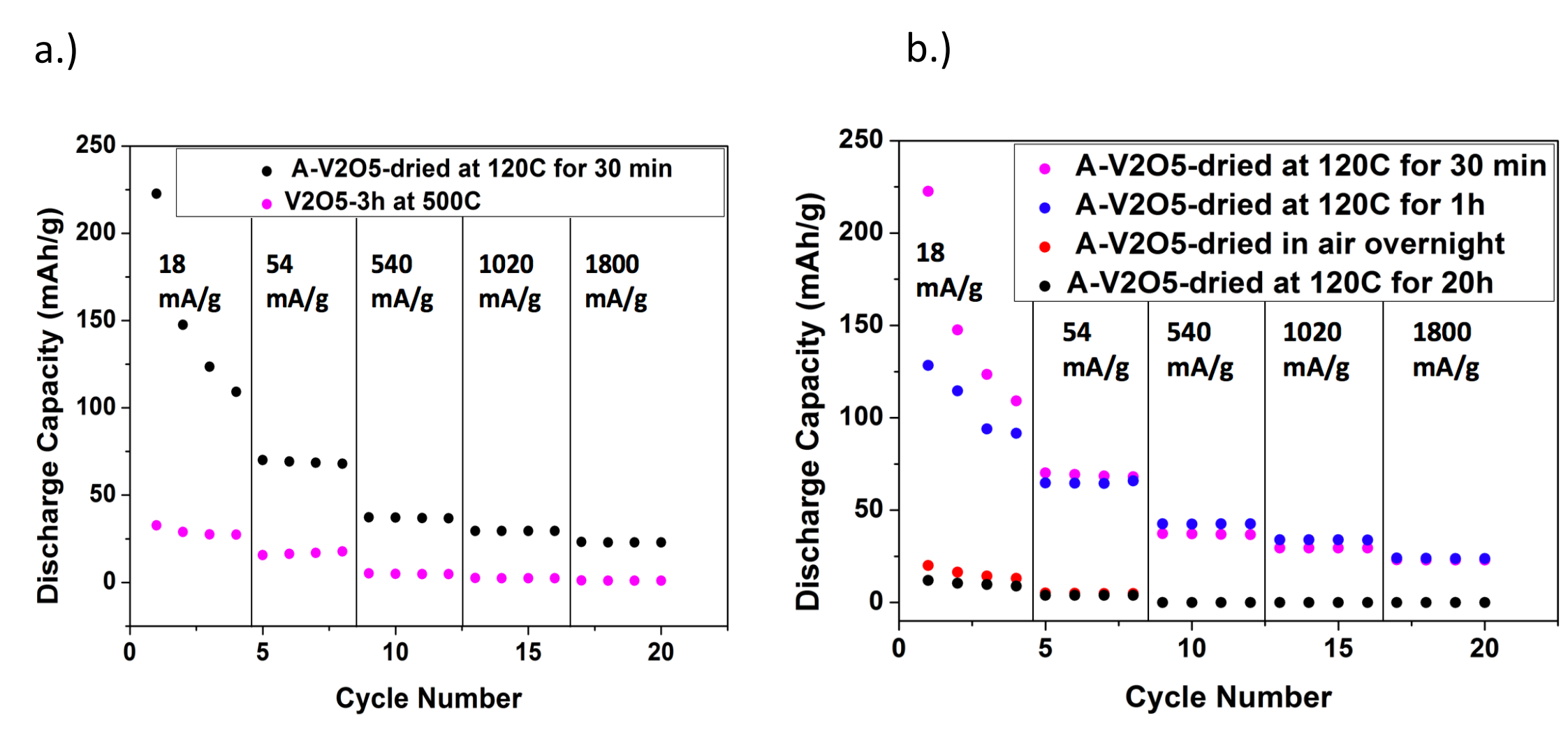

a.) Rate Performance Data for Amorphous and Crystalline V2O5 cycled in $0.1 \mathrm{M} \mathrm{Mg}\left(\mathrm{ClO}_{4}\right)_{2}{ }^{*} 6 \mathrm{H}_{2} \mathrm{O}$. Amorphous $\mathrm{V} 2 \mathrm{O} 5$ outperforms crystalline V2O5 at every current density. b.) Rate Performance Data for Amorphous V2O5 samples with variable drying protocols. Increasing drying time results in lower capacity, which suggests that structural water content is crucial for mg-ion insertion into electrodeposited V205 thin films.

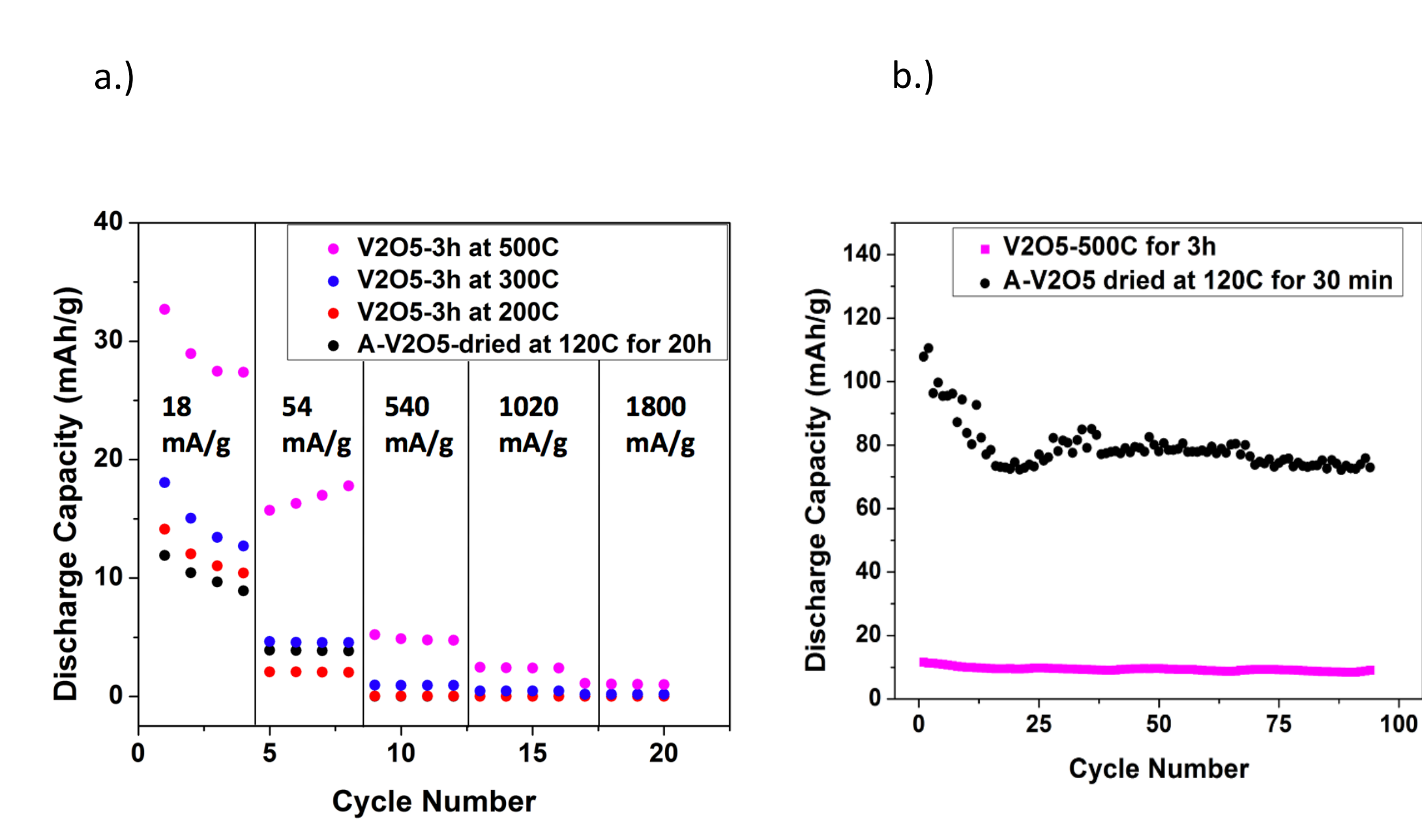

a.) Comparison of rate performance of amorphous V2O5 dried at $120 \mathrm{C}$ for 20 hours with annealed V2O5 samples. All samples were cycled in $0.1 \mathrm{M} \mathrm{Mg}\left(\mathrm{ClO}_{4}\right)_{2} * 6 \mathrm{H}_{2} \mathrm{O}$. b.) Long term cycling $120 \mathrm{C}$ for 30 minutes and a $\mathrm{V}_{2} \mathrm{O}_{5}$ thin film annealed at $500^{\circ} \mathrm{C}$ for 3 hours.
Conclusion and Future Studies

In recent studies, there has been discrepancy as to whether amorphous or crystalline $\mathrm{V} 2 \mathrm{O} 5$ offers superior $\mathrm{mg}$-ion storage capabilities. The aim structures in order to determine a link between crystallinity and electrochemical performance. As supported by the comparative rate performances of dry and wet amorphous samples, structural water is crucial for achieving high capacity magnesium-ion storage enhances mg-ion insertion into V2O5 for three reasons. Structural water may contribute to charge shielding of $\mathrm{Mg} 2+$ species, and increase
void spacing for multivalent ion insertion. Additionally increased water void spacing for multivalent ion insertion. Additionally, increased water
content may lead to higher capacity attributable to increased levels of proton insertion. At this point, we cannot conclusively report on the impact of structure, although it appears that there is no significant film samples. Thus, we postulate that in the case of electrodeposited thin films, the advantage comes from the water that is integrated into the thin film structure during deposition. Although the role of water in promoting magnesium-ion insertion into $\mathrm{V} 2 \mathrm{O} 5$ is well studied, it must also be carefully considered when evaluating amorphous and crystalline $\mathrm{V}_{2} \mathrm{O}_{5}$ structures prepared via aqueous deposition methods, as electrochemical performance will change significantly depending on the extent of structural hydration.

Future work will include ICP-MS analysis in order to quantify the . experiments. Furthermore, ICP-MS analysis will give an indication of how much of the capacity is attributable to proton insertion rather than magnesium insertion. In addition to ICP-MS, we will perform thermogravimetric analysis in order to more clearly relate drying protocols with water loss. This way, we will be able to distinguish the effects of loosely bound surface water and more tightly bound lattice water on thin film capacity. Lastly, in order to study the effect of V2O5 cystallinity independent of structural water content, we would like to compare the electrochemical performance of amorphous and crystalline V2O5 prepared from ultrahigh vacuum deposition processes,
such as atomic layer deposition (ALD).

\section{References}

Yoo, H. D.; Shterenberg, I.; Gofer, Y.; Gershinsky, G.; Pour, N.; Aurbach, D., Mg rechargeable batteries: an on-going challenge. Energy \& Environmental Science 2013, 6 (8), 2265-2279.

Wang, F.; Fan, X.; Gao, T.; Sun, W.; Ma, Z.; Yang, C.; Han, F.; Xu, K. Wang, C., High-Voltage Aqueous Magnesium Ion Batteries. Acs Central Science 2017, 3 (10), 1121-1128.

Kim, D. \& Ryu, Amorphous $\mathrm{V}_{2} \mathrm{O}_{5}$ Positive Electrode Materials by Erecipitation Method in Magnesium Rechargeab

Acknowledgements

(i) ENERTCY Basic Energy Sciences AMLAB 